Asian J. Med. Biol. Res. 2018, 4 (4), 337-342; doi: 10.3329/ajmbr.v4i4.40105

\author{
Asian Journal of \\ Medical and Biological Research \\ ISSN 2411-4472 (Print) 2412-5571 (Online) \\ www.ebupress.com/journal/ajmbr
}

\title{
Article \\ A retrospective study investigating the effects of outpatient physiotherapy on lower back pain in Bangladesh
}

\author{
Muhammad Shahidul Islam ${ }^{1}$ and Firoz Ahmed Mamin ${ }^{2 *}$ \\ ${ }^{1}$ Department of Physiotherapy, SAIC Institute of Medical Technology, Mirpur, Dhaka, Bangladesh \\ ${ }^{2}$ Department of Rehabilitation Science, Bangladesh Health Professions Institute (BHPI). CRP, Savar, Dhaka, \\ Bangladesh
}

*Corresponding author: Firoz Ahmed Mamin, Department of Rehabilitation Science, Bangladesh Health Professions Institute (BHPI). CRP, Savar, Dhaka, Bangladesh. Phone: +8801776655081; E-mail: mamincns@gamil.com

Received: 11 October 2018/Accepted: 27 October 2018/ Published: 30 December 2018

\begin{abstract}
Low Back Pain (LBP) is a widespread global health problem. Almost $80 \%$ of people suffer from this condition at some point in their life. Management of LBP is a challenge for health care professionals. Out of all management strategies Physiotherapy is commonly and widely used around the globe for treating patients with LBP.A large number of people seek physiotherapy intervention for their LBP in Bangladesh however, studies regarding their treatment outcomes following physiotherapy are not available. This study was a retrospective, descriptive study of patients' with Chronic Low Back Pain (CLBP) attending physiotherapy. The objective of this study was to investigate the level of pain and functional limitation following physiotherapy intervention. Information regarding 1,000 patients with LBP was retrieved from patient records in the Physiotherapy department at the Centre for the Rehabilitation of the Paralysed (CRP). Outcomes investigating pain and function were recorded and analysed. 63\% of all participants were female. Pain was significantly reduced among patients with CLBP after receiving physiotherapy. Extension and flexion of the lumbar spine were lost in about $80 \%$ and $56 \%$ of patients respectively. Movements of the lumbar spine were improved after physiotherapy. Nearly $50 \%$ of patients reported improvements of more than $75 \%$ after receiving physiotherapy for LBP. Patients with CLBP had different characteristics. In this study it was not possible to record all necessary information. In general most of the patients' outcomes were improved after receiving physiotherapy intervention.
\end{abstract}

Keywords: low back pain; physiotherapy; functional outcome

\section{Introduction}

Low back pain (LBP) is one of the most common musculoskeletal problems around the world, affecting about $80 \%$ of people in their lifetime (Gellhorn et al., 2010). LBP is known globally as a prime contributor to Years Lived with Disability (YLDs) (Robert et al., 2014) and it creates a substantial personal, community, and financial burden (Dionne et al., 2006). Himalowa and Frantz (2012) stated that day-by-day the impacts of LBP on the economic, social and public health situation are increasing enormously.

Of those who suffer with acute LBP, $75-90 \%$ of them recover within 6 weeks regardless of medical intervention, however up to $25 \%$ are at risk of developing chronic pain and disability (Benjamin et al., 2014). People with CLBP are more likely to seek care and use more health care services; for this reason there is an increased demand for prescription medication and visits to physicians, physiotherapists and chiropractors (Freburger et al., 2009). Currently LBP is treated mainly with analgesics and alternative treatments such as physiotherapy, rehabilitation and spinal manipulation. Disc surgery remains the last option when all other strategies fail (Phillips, 2013) however; Physiotherapy intervention is the most effective amongst all other 
conservative management in LBP (Lau, 2008). In clinical practice different practitioners utilise a range of physiotherapy treatment strategies with varying degrees of effectiveness. These include joint mobilization, manipulation, exercise therapy, soft tissue massage techniques, electrotherapy, and traction (Dunsford, 2011).

The outcome of physiotherapy on patients with LBP has been assessed in various countries across different settings. In Bangladesh, around 2,000 physiotherapists are currently working and it is estimated that most of them are working in musculoskeletal field and treating patients with LBP. The outcome of physiotherapy intervention on LBP in Bangladesh is still unknown. The primary purpose of this study is to investigate the outcome of physiotherapy on LBP.

\section{Materials and Methods}

\subsection{Study design}

This study was a retrospective, descriptive study conducted in the Physiotherapy Department based at the Centre for the Rehabilitation of the Paralysed (CRP), Savar, Dhaka. CRP is one of the biggest rehabilitation and physiotherapy centers in Bangladesh, treating hundreds of patient with LBP every day. Participants' data was obtained from the Physiotherapy Department database.

\subsection{Data Source}

Patient documentation was collected and stored at the department.1100 paper patient records were randomly selected for analysis using simple randomization generated by file numbers. Information was collected from patients who attended physiotherapy for their LBP between $1^{\text {st }}$ January 2015 and $31^{\text {st }}$ December 2015.100 records were excluded due to incomplete records. A power calculation was used to ensure a large enough sample size was collected to determine the true significance of the results, it was decided that a larger sample size would be used in order to obtain a good representation of physiotherapy intervention on those with LBP.

The multidisciplinary team considered patients with a history of Non Specific CLBP for more than three months. There were no restrictions on age or gender in this study. Patients whose LBP was caused by a particular disease or who had undergone any surgical intervention for their LBP were excluded.

Ethical permission was obtained from the Institutional Review Board, at Bangladesh Health Professions Institution (BHPI) prior to this study.

\subsection{Measurements}

Demographic information including age and sex was identified. Information on the cause of pain, past medical history, loss of movement and history of pain medication was collected. Pain was recorded before and after the intervention, in the patients' record, using the Visual Analogue Scale (VAS). An additional subjective measure was taken from patients about their pain, based on a 4 point Likert scale. This scale was divided in 4 sections based on their perceived improvement: 1 to $25 \%, 26$ to $50 \%, 51$ to $75 \%$ and 76 to $100 \%$. Patients had choices to put tick marks on any options based on their opinion. Patients had the choice to select the most appropriate category that reflected their improvements in pain.

\subsection{Data analysis}

Descriptive statistics were used to portray the data and the paired $t$ test to evaluate the difference between pre and post intervention outcomes. Differences were considered significant if $\mathrm{p}<0.05$.Data analysis was conducted using the Statistical Package for the Social Science (SPSS) version 19.

\section{Results}

\subsection{Patient Demographics and Health Information}

1,000 patient records were retrieved for this study, of which $37.5 \%$ were male. The mean age of the patients was 40 years. The majority of the patients were 21-30 years old. Approximately $88 \%$ of patients were unaware of the cause of their pain. The majority of patients had a moderate loss of lumbar spine extension and $65 \%$ of patients had axial pain. About $45 \%$ of patients were on pain medication for their LBP. Most patients scored their pain between 5 and 8 on the VAS at baseline. Please see Table 1 for further details. 
Table 1. Patient demographics and health information.

\begin{tabular}{llll}
\hline Variables & Categories & Frequency $(\mathbf{N}=\mathbf{1 0 0 0})$ & Percentage \\
\hline \multirow{2}{*}{ Gender } & Male & 376 & 37.6 \\
& Female & 624 & 62.4 \\
Age(Years) & $<20$ & 278 & 27.8 \\
& $21-30$ & 527 & 52.7 \\
Causes of Pain & $31-40$ & 186 & 18.6 \\
& $41-50$ & 9 & .9 \\
Past Medical History & Unknown & 878 & 87.8 \\
& Trauma & 122 & 12.2 \\
Drug History & DM & 135 & 13.5 \\
& HTN & 184 & 18.4 \\
& Heart Disease & 23 & 2.3 \\
Pain Characteristics & Pain medication & 453 & 45.3 \\
& Axial & 656 & 65.6 \\
& Unilateral above knee & 83 & 8.3 \\
& Unilateral below knee & 147 & 14.7 \\
& Bilateral & 72 & 7.2 \\
& Not assessed & 42 & 4.2 \\
Pain Intensity & $1-3$ & 10 & 1.1 \\
(VAS scale) & 4 & 33 & 3.3 \\
& 5 & 210 & 21.0 \\
& 6 & 244 & 24.4 \\
& 7 & 197 & 19.7 \\
& 8 & 218 & 21.8 \\
& 9 & 50 & 5.0 \\
& 10 & 18 & 1.8 \\
\hline
\end{tabular}

DM=Diabetes Mellitus, HTN=Hypertension, VAS=Visual Analogue Scale

\subsection{Physiotherapy intervention}

Most patients had multiple sessions of physiotherapy, with 15 to $53 \%$ receiving 6 to 15 sessions. Most of the patients received a range of physiotherapy interventions including therapeutic exercise and manual therapy. Please see Table 2 for details.

Table 2. Types of physiotherapy intervention and number of treatment sessions attended.

\begin{tabular}{llll}
\hline Variables & Categories & Frequency & Percentage \\
\hline \multirow{3}{*}{ Types of Physiotherapy } & Therapeutic exercise & 827 & 82.7 \\
intervention & Manual therapy & 969 & 96.9 \\
& Electrotherapy & 884 & 88.4 \\
& Home advice & 957 & 95.7 \\
& $1-5$ & 156 & 15.6 \\
Number of Sessions & $6-10$ & 277 & 27.7 \\
& $11-15$ & 252 & 25.2 \\
& $16-20$ & 94 & 9.4 \\
& $21-25$ & 66 & 6.6 \\
& $26-30$ & 75 & 7.5 \\
\end{tabular}

\subsection{Improvement on pain and function after receiving physiotherapy intervention}

This study found that pain was significantly reduced after receiving physiotherapy intervention. At base line the mean pain score was 6.64 on the VAS scale whereas at discharge it was 3.08. Post physiotherapy intervention the patients' quality of movement was improved. Post intervention, most patients had a greater restriction into lumbar extension compared to lumbar flexion. Please see Table 3 for details. 
Table 3. Pain and lumbar spine range of movement before and after physiotherapy.

\begin{tabular}{llll}
\hline Variable & Before physiotherapy & After physiotherapy & $\boldsymbol{p}$ value \\
\hline $\begin{array}{l}\text { Intensity of Pain } \\
\text { (VAS scale) }\end{array}$ & $6.64+-1.54$ (Mean+-SD) & $3.08+4.56$ (Mean+-SD) & .00 \\
$\begin{array}{l}\text { Lumbar flexion } \\
\quad \text { Major loss }\end{array}$ & $5.3 \%$ & & \\
$\quad$ Moderate loss & $19.1 \%$ & $.4 \%$ & .05 \\
$\quad$ Mild loss & $31.1 \%$ & $2.1 \%$ & .00 \\
Lumbar extension & & $6.1 \%$ & .00 \\
$\quad$ Major loss & $9.6 \%$ & $1.3 \%$ & .00 \\
$\quad$ Moderate loss & $41.9 \%$ & $4.3 \%$ & .00 \\
$\quad$ Mild loss & $28 \%$ & $8.6 \%$ & .00 \\
\hline
\end{tabular}

$p$ - value derived from paired $t$ test.

\subsection{Self reported outcomes}

About $50 \%$ of patients reported a reduction of up to $70 \%$ in their levels of pain. $21 \%$ of patients reported a reduction of 50 to $74 \%$ and only $19 \%$ of patients reported their pain reduced less than $25 \%$. Please see Table 4 for more information.

Table 4. Self-reported improvement in pain reported post physiotherapy intervention.

\begin{tabular}{lccc}
\hline Variable & Categories & Frequency & Percentage \\
\hline \multirow{3}{*}{ Overall improvement } & $25 \%$ or less & 188 & 18.8 \\
& $26 \%-50 \%$ & 106 & 10.6 \\
& $51 \%-75 \%$ & 210 & 21.0 \\
& $76 \%$ or more & 496 & 49.6 \\
\hline
\end{tabular}

\section{Discussion}

This study was conducted at Physiotherapy department of CRP. A total 1000 patient' records were assessed for this retrospective study. This study found more female patients attended CRP for management of their LBP. This study included 624 were female patients and only 376 were male, which may suggest that females are more prone to developing LBP than males. Schneider et al. (2006) found that the prevalence of LBP was greater in women than in men. They studied 1,900 participants, 1,037 of which were women. Similarly a literature review by Schneider et al. (2005) highlighted that in seven out of twelve studies, there was a significantly higher prevalence of pain in women. The mean age of the participants was 40 years old, with the most affected age group between 31-50 years old. Xu et al. (2012) found a similar mean age 37 years among patients with LBP. Dionne et al. (2006) stated that age is one of the most common factors in the development of LBP with the highest incidence of LBP observed in the third decade of life. In addition, Casazza (2012) explained that LBP most commonly begins between 20 and 40 years of age although LBP can occur at any age.

About $87.7 \%$ of patients stated that they did not find any reasonable cause for their pain, with only $12.3 \%$ noting trauma as a possible cause of their LBP. Most LBP has no specific cause and is therefore termed as non-specific LBP (Hartvigsen et al., 2018). Our study supports this theory that LBP rarely occurs due to a specific identifiable reason. The duration of LBP may vary from one day to many years. In this study the mean duration of pain was 11 months. Approximately $65.5 \%$ of subjects experienced axial back pain. About $8.3 \%$ had unilateral pain above their knee and $14.8 \%$ had unilateral pain below the knee.

After receiving physiotherapy intervention pain was significantly reduced. At base line the mean score of pain was 6.64 and during discharge it was about 3.08 on the VAS scale. This study found that physiotherapy intervention significantly improves the symptoms of LBP. Our primary objective of this study was to investigate the improvement in pain following physiotherapy, as pain was the main complaint of patients with LBP seeking help from health care professionals. In addition to pain, spinal movement was reduced in a large number of patients with about $80 \%$ experiencing a decrease in lumbar extension compared to $56 \%$ losing range into lumbar flexion. Post physiotherapy management spinal movement improved, only $15 \%$ of patients had reduced lumbar extension compared to $9 \%$ of patients with lumbar flexion loss. Physiotherapy has proven to be an effective management strategy for CLBP in various previous studies (Haydenet et al., 2005). Physiotherapy is effective in improving quality of life for patients with LBP (Cairns, 2006). Gracey (2002), suggested that physiotherapy can improve function in patients with LBP be it acute, sub-acute or chronic. 
On discharge patients provided subjective feedback about their improvement in their pain. About $18.8 \%$ of subjects rated their improvement $25 \%$ or less, $29.4 \%$ had improvement between $26 \%$ and $50 \% .497$ subjects had improvement as $75 \%$ or more.

This study has several limitations. Data was obtained from a single centre therefore it may be difficult to assume it is represents the rest of the population. Many of patients' assessment forms were not completed and so it was not possible to collect all relevant information. The scale used to measure self-reported improvement in pain grouped together patients who reported an improvement of $25 \%$ or less. It is therefore difficult to decipher between the patients who showed no improvement and minor improvements following physiotherapy. However, this only represented $18.8 \%$ of the study sample and an overwhelming majority $(81.2 \%)$ reported an improvement of $26 \%$ or more. About $45 \%$ of patients took some form of analgesia during their physiotherapy treatment; therefore it is difficult to determine the true cause of their improvements in pain.

\section{Conclusion}

LBP is a multifaceted problem and managed in various ways. Physiotherapy intervention can be successfully utilized for this patient group, improving pain and mobility of the spine. This study supports the use of physiotherapy to treat patients with CLBP, demonstrating significant improvements in LBP and lumbar range of motion. Findings from this study will guide further research in this field, a prospective multicenter study investigating the effects of physiotherapy on LBP is recommended.

\section{Acknowledgements}

Authors would like to thank Department of Physiotherapy, CRP, Savar, for permitting data collection.

\section{Conflict of interest}

None to declare.

\section{References}

Benjamin H, D Christine, H Toby, M Philippe and N Henri, 2014. The efficacy of manual therapy and exercise for different stages of non-specific low back pain: an update of systematic reviews. J. Man. Manip. Ther., 22: 60.

Cairns MC, NE Foster and C Wright, 2006. Randomized controlled trial of specific spinal stabilization exercises and conventional physiotherapy for recurrent low back pain. Spine, 31: 670-681.

Casazza BA, 2012. Diagnosis and treatment of acute low back pain. Am. Fam. Physician, 85: 343-350.

Dionne CE, KM Dunn and PR Croft, 2006. Does back pain prevalence really decrease with increasing age? A systematic review. Age and Ageing, 35: 229-234.

Dunsford A, S Kumar and S Clarke, 2011. Integrating evidence into practice: use of McKenzie-based treatment for mechanical low back pain. J. Multidiscip. Healthc., 4: 393.

Freburger JK, GM Holmes, RP Agans, AM Jackman, JD Darter, AS Wallace, LD Castel, WD Kalsbeek and TS Carey, 2009. The rising prevalence of chronic low back pain. Arch. Intern. Med., 169: 251-258.

Frost H, SE Lamb, HA Doll, PT Carver and S Stewart-Brown, 2004. Randomised controlled trial of physiotherapy compared with advice for low back pain. Br. Med. J., 329: 708.

Gellhorn AC, L Chan, B Martin and J Friedly, 2010. Management patterns in acute low back pain. Spine, 37:775-782.

Gracey JH, SM McDonough and GD Baxter, 2002. Physiotherapy management of low back pain: a survey of current practice in Northern Ireland. Spine, 27: 406-411.

Hartvigsen J, J Mark, Q Alice Kongsted, LF Manuela, G Stéphane, H Damian, JaroKarppinen, P Glenn, Smeets and U Martin, 2018. What low back pain is and why we need to pay attention. The Lancet, March 21, doi.org/10.1016/ S0140-6736(18)30480-X

Hayden J, MW vanTulder, A Malmivaara and BW Koes, 2005. Exercise therapy for treatment of non- specific low back pain. Cochrane Database Syst. Rev., Issue 3. Art. No.: CD000335. DOI: 10.1002/14651858.CD000335.pub2.

Himalowa S and J Frantz, 2012. The effect of occupationally- related low back pain on functional activities among male manual workers in a construction company in Cape Town, South Africa. Occupational Health Southern Africa, 18: 4.

Lau PMY, DHK Chow and MH Pope, 2008. Early physiotherapy intervention in an Accident and Emergency Department reduces pain and improves satisfaction for patients with acute low back pain: a randomised trial. Aust. J. Physiother., 54: 243-249. 
Phillips FM, PJ Slosar, JA Youssef, G Andersson and F Papatheofanis, 2013. Lumbar spine fusion for chronic low back pain due to degenerative disc disease: a systematic review. Spine, 38: 409-422.

Robert F, P Sue, E Sandra, S Clive, P Tamar, R Dévan, F Christian and U Martin, 2014. A systematic review and meta-synthesis of the impact of low back pain on people's lives. BMC Musculoskeletal Disorders, 15: 50 .

Schneider S, D Randol and M Buchner, 2006. Why do women have back pain more than man? A representative prevalence study in the federal republic in German. Clin. J. Pain, 22: 738-747.

Schneider S, H Schmitt, S Zoller and M Schiltenwolf, 2005. Workplace stress, lifestyle and social factors as correlates of back pain: a representative study of the German working population. Int. Arch. Occup. Environ. Health, 78: 253-269.

Xu G, D Pang, F Liu, D Pei, S Wang and L Li, 2012. Prevalence of low back pain and associated occupational factors among Chinese coal miners. BMC Public Health, 12: 149. 